\title{
Offrez des calories pour la vie!
}

\section{Marc Subilia}

Pasteur et médecin

\section{Une situation paradoxale}

Pendant 15 ans, j’ai étudié puis pratiqué la médecine. Puis, au terme d'études de théologie, j'ai été pendant 29 ans pasteur dans des paroisses de l'Eglise réformée du canton de Vaud (où j'accomplis actuellement un remplacement en aumônerie des EMS).

Durant toutes ces années, une situation paradoxale m'a paru de plus en plus choquante. Voyez: D’un côté, la publicité des médias propose une surenchère de moyens, plus ou moins coûteux, pour se défaire des calories accumulées en excès. En Suisse, par exemple, près de la moitié de la population souffre actuellement d'un excès de poids. Il existe certes des maladies où l'excès pondéral est indépendant de l'alimentation, mais dans la plupart des cas le surpoids est dû à une alimentation trop riche en calories.

Nourriture trop abondante, parfois mal équilibrée, multiples petits repas entre les repas, etc., le résultat est le même: le pèse-personne indique l'excès, et il s'agit dès lors de tenter de brûler au plus vite ces calories superflues et gênantes! Trop de calories alourdissent le corps et le déforment, fatiguent le cœur, usent les articulations...

De l'autre côté, des personnes en sont réduites à se nourrir d'herbes et de racines, dans une situation de famine qui les conduit inexorablement à la mort, au terme d'un cruel et long supplice. Ces hommes, ces femmes, ces enfants sont littéralement condamnés à mourir de faim. Cette situation, paradoxale et scandaleuse, n'est pourtant pas inéluctable.

Voici une proposition concrète, développée dans une brochure qui vient de paraître (voir les informations pratiques).

\section{Offrez des calories!}

Concrètement, il s'agit de renoncer à un repas par semaine, de préférence un souper, pour en offrir la contre-valeur à une œuvre de votre choix luttant contre la faim.
Il est vrai que la simple idée de restreindre l'apport calorique, même modestement, évoque un manque, une privation, une frustration pénible. L'idée même du jeûne paraît si déplaisante qu'on juge cette pratique périmée, décalée, pour y échapper. Alors même qu'elle est prescrite dans des religions et dans le monde des soins depuis le fond des âges.

Mais il est vrai aussi que, lorsqu'on offre librement un cadeau à quelqu'un, on ne se sent ni lésé ni frustré! Le donateur se réjouit de ce qu'il ne considère pas comme une perte pénible, mais comme une source de joie pour celui qui reçoit le cadeau... et pour lui-même.

Par analogie, celui qui donne son sang supporte le désagrément que cela lui cause, parce qu'il se réjouit de ce que son don va soulager celui qui le reçoit: il ne s'agit pas d'une perte de sang, mais d'un don qui parfois peut sauver une vie.

\section{Une expérience bénéfique}

Quand je renonce à un repas du soir, je vais donc me coucher le ventre creux. Sensation désagréable, mon estomac proteste, des gargouillis se font entendre... Mes entrailles ont peur de manquer et réclament leur dû! Mais, à y regarder de plus près, il s'agit moins de faim que d'envie de manger. Mon organisme se passe volontiers de nourriture pendant quelques heures. Mon sentiment de frustration est très passager, puisque bientôt je vais de nouveau pouvoir manger à satiété. Il me permet toutefois, de manière légère et éphémère, de compatir avec ceux qui n'auront à manger ni demain, ni aprèsdemain, ni les jours suivants.

Du coup, je ressens comme un scandale intolérable que, sur cette terre qui regorge de biens, tant de personnes puissent être torturées par la faim jusqu'à ce que mort s'en suive. La lutte contre la faim s'impose avec évidence comme prioritaire.

En donnant l'équivalent de mon repas à une des œuvres luttant contre la faim, je ne donnerai pas, comme je le 


\section{Informations pratiques}

La brochure "Offrez des calories pour la vie!» décrit la démarche proposée. Elle peut être commandée sur le site www. descaloriespourlavie.ch ou par courrier postal au prix de $6 \mathrm{CHF}$ port compris. Un bulletin de versement sera joint à l'envoi. Offrez la somme économisée, à votre rythme, à une œuvre qui vous tient à cœur, et qui lutte contre la faim par des secours en cas de détresse, des soins, la prévention, l'éducation, le développement, etc. (la brochure mentionne 4 œuvres dignes de confiance, à titre indicatif).

Pour nous contacter: info[at]descaloriespourlavie.ch

fais souvent, une partie de mon superflu pour secourir mon prochain. Je fais l'expérience (si limitée soit-elle) de partager ce qui est nécessaire à la vie (ma nourriture) avec celui qui est menacé de mort. J'éprouve une réelle sympathie (= souffrance avec). En offrant ces calories, que je renonce à manger, à qui en manque, je ressens une joie intérieure.

D'autre part, en buvant de l'eau, de la simple eau du robinet, en lieu et place de mon repas, je prends mieux conscience que c'est un privilège d'avoir une eau claire, sans goût déplaisant, d'une fraîcheur agréable, et surtout propre et sûre. Je me délecte de cette eau cristalline qui est là, à portée de main, sans restriction...

Je me souviens alors que tant de personnes n'ont accès qu'à une eau trouble, insalubre, source de graves maladies. Et parfois sont complètement privées d'eau. Je suis alors plus vivement conscient de la nécessité urgente, impérieuse, de partager avec mes frères et sœurs humains cet élément précieux indispensable à la vie. Ce qui peut se faire, par exemple, en favorisant l'accès à des points d'eau simples et efficaces.

\section{Les jours suivants...}

Le lendemain d'un souper auquel j'ai renoncé, bonne surprise! Je ne suis pas plus affamé qu'un autre jour, au petit déjeuner, et j'ai la satisfaction de me sentir dispos et alerte, le corps et le cœur légers.

Les jours suivants, je deviens plus conscient de ce que j'ingère. Je reprends le contrôle de certaines habitudes automatiques, de grignotages incessants entre les repas, etc. J'ai réussi à surmonter ma peur de manquer. Je suis peu à peu libéré de cette stratégie inadéquate et tyrannique qui prétend combler toutes les frustrations, déceptions et angoisses de la vie par un remplissage alimentaire excessif.

Par ailleurs, je savoure bien davantage ma nourriture, appréciant ce qui est dans mon assiette comme un privilège. Mes repas sont source de reconnaissance puisque je me rappelle que la nourriture est précieuse, et qu'elle ne va pas de soi.

\section{Rêvons un peu...}

Si vous décidez de sauter dorénavant un repas par semaine et d'offrir la somme équivalente à la lutte contre la faim, et que vous persuadez, durant le premier mois qui suit votre décision, deux personnes de faire de même; si donc chacune de ces deux personnes, durant le premier mois où elle a accepté de sauter un repas par semaine, en entraîne deux autres à faire de même, et ainsi de suite, quelles en seraient les conséquences? $\mathrm{Au}$ bout de 12 mois, environ 4000 personnes (voire même familles, suivant les conditions de vie) seraient sauvées de la famine; 16 millions au bout de 24 mois; et plus de 4 milliards après 34 mois! Bref, en moins de 3 ans, plus personne ne mourrait de faim sur cette terre! Et cela sans augmentation de budget! Sans que personne ne sorte de son porte-monnaie un franc de plus que d'ordinaire... mais par un modeste don de calories!

\section{Soyons réalistes!}

Si, en offrant la contre-valeur d'un souper par semaine, vous sauvez la vie d'une personne (voire d'une famille) qui sans cela serait morte de faim, qu'est-ce qui empêche de commencer tout de suite? Quel bonheur de pouvoir entreprendre quelque chose de concret sans dépendre de décisions gouvernementales, de l'inertie de diverses instances... Sans devoir rester les bras ballants devant les malheurs du monde!

Bien sûr, il y a mille autres manières de combattre les souffrances du monde, ses injustices et ses violences. Celle-ci en est une qui ne dépend que de nous-mêmes, qui n'a pas d'effet sur notre budget (parfois précaire), qui peut être mise en œuvre immédiatement... et qui sauve des vies.

\section{Le bonheur des uns... fait le bonheur des autres!}

En offrant ces calories que vous renoncez à ingérer à qui en manque cruellement, vous expérimentez en profondeur la joie du partage. Cette joie intérieure se renouvelle de semaine en semaine, c'est ce qui permet de tenir dans la durée.

Votre corps, souvent éprouvé par un apport calorique excessif, appréciera l'épargne hebdomadaire de ces quelques calories superflues. Vous en constaterez bientôt les heureux effets sur tout votre organisme. Tout à la fois soulager les souffrances d'un affamé et se porter mieux, c'est faire l'expérience renouvelée que le bonheur des uns fait ici le bonheur des autres.

Ainsi donc, nous vous invitons à offrir, vous aussi, des calories pour la vie! 